\title{
Generation of CRISPR/Cas9-mediated lactoferrin-targeted mice by pronuclear injection of plasmid pX330
}

\author{
Mengxu GE ${ }^{1 *}$, Fei LIU ${ }^{2 *}$, Fei CHANG ${ }^{1}$, Zhaolin SUN ${ }^{1}$, Jing FEI ${ }^{1}$, Ying GUO ${ }^{1}$, Yunping DAI ${ }^{1}$, Zhengquan YU ${ }^{1}$, \\ Yaofeng ZHAO ${ }^{1}$, Ning LI (凶) $)^{1,2}$, Qingyong MENG $(\bowtie)^{1}$
}

1 State Key Laboratory of Agrobiotechnology, College of Biological Science, China Agricultural University, Beijing 100193, China

2 College of Animal Science and Technology, Yunnan Agricultural University, Kunming 650201, China

\begin{abstract}
Lactoferrin is a member of the transferrin family of multifunctional iron binding glycoproteins. While numerous physiological functions have been described for lactoferrin, the mechanisms underlying these functions are not clear. To further study the functions and mechanisms of lactoferrin, we modified the lactoferrin promoter of mice using the CRISPR/Cas9 system to reduce or eliminate lactoferrin expression. Seven mice with lactoferrin promoter mutations were obtained with an efficiency of $24 \%(7 / 29)$ by injecting the plasmid pX330, expressing a small guide RNA and human codonoptimized SpCas9, into fertilized eggs of mice. Plasmid integration and off-targeting of pX330 were not detected. These results confirmed that pronuclear injection of a circular plasmid is a feasible and efficient method for targeted mutagenesis in mice.
\end{abstract}

Keywords lactoferrin, promoter, CRISPR/Cas9, plasmid pX330

\section{Introduction}

Lactoferrin (LF) is a multifunctional iron binding glycoprotein belonging to the transferrin family, with a molecular weight of $80 \mathrm{kDa}$. In 1938, LF was first identified as a red protein that was present in bovine milk $^{[1]}$. In 1960, it was isolated from human milk by Groves and was subsequently recognized as a major iron binding protein ${ }^{[2-4]}$. LF is not only present in milk and

Received April 12, 2015; accepted June 4, 2015

Correspondences: ninglcau@cau.edu.cn, qymeng@cau.edu.cn

${ }^{*}$ These authors contribute equally to the work several other exocrine secretions, but it is also present in the secondary granules of neutrophils. Several biological functions have been described for LF, including iron homeostasis, immunomodulation, cellular growth and differentiation, antimicrobial, antioxidant, anti-inflammatory, antiviral and anticancer properties ${ }^{[5-14]}$. However, the mechanisms underlying these functions are not clear. In this study, we aimed to obtain mice in which LF expression was reduced or eliminated, thereby providing a model to study the functions and mechanisms of LF.

Of the next-generation genome editing technologies, the CRISPR (clustered regularly interspaced short palindromic repeats)/Cas (CRISPR-associated) system has been demonstrated to be a robust tool for genome engineering. The CRISPR/Cas system is an RNA-based adaptive immune system in bacteria and archaea that can destroy invasive plasmids, phages and viruses ${ }^{[15-17]}$. Compared with the complex and time-consuming design and generation of zinc finger nucleases and transcription activator-like effector nucleases, the CRISPR/Cas geneediting approach only requires Cas 9 and small guide RNAs (sgRNAs). Because of its simplicity, low cost and high efficiency, the CRISPR/Cas system has become a widely used platform for genome editing in animals and plants ${ }^{[18]}$. Recently, the type II CRISPR/Cas system has been demonstrated to be an efficient gene-editing technology that has been used successfully to produce many gene-targeted cell lines and animals ${ }^{[19-24]}$.

In this study, we injected plasmid pX330 expressing an sgRNA and human codon-optimized SpCas9 (hSpCas9) into mouse zygotes to modify the mouse $L F$ promoter, and we obtained $L F$ gene promoter mutation mice without detectable random plasmid integration and off-targeting. These mutated mice can provide a model for studying the functions and mechanisms of LF. 


\section{Materials and methods}

\subsection{Animals}

Kunming mice used in this project were bought from the Chinese Experimental Animal Resources Research Institute for Food and Drug Control. Animals were handled according to the Guidelines for the Care and Use of Laboratory Animals established by the Beijing Association for Laboratory Animal Science. Animal experiments were approved by the Animal Ethics Committee of the College of Biological Sciences, China Agricultural University.

\section{2 pX330-sgRNA and 2Se-sgRNA plasmid construction}

The targeting sites were designed in the basic $L F$ promoter areas using the free website (http://crispr.mit.edu). We synthesized oligonucleotides that were complementary to the targeting sites with the three highest scores (Table 1). The polymerase chain reaction (PCR) primers used in this work are shown in Table S1 (Appendix A). Six pairs of oligonucleotides were designed for three target sites. Three pairs of oligonucleotides (C-mRJM-1-1-F/R, C-mRJM-12-F/R, C-mRJM-1-3-F/R) were annealed to doublestranded DNA and then individually ligated to the $B b s$ I sites of plasmid pX330 (authorized by Dr. Feng Zhang from the Massachusetts Institute of Technology and obtained from Dr. Bo Zhang at the College of Life Sciences, Peking University) to construct three pX330sgRNA plasmids. The other three pairs of oligos (DCmRJM-1-1-F/R, DC-mRJM-1-2-F/R, DC-mRJM-1-3-F/R) were individually ligated into the EcoRI and BamHI (New England Biolabs, Ipswich, MA, USA) sites of the dualcolor reporter vector $2 \mathrm{Se}$ (which was constructed in our laboratory). Then, the pX330-sgRNA and 2Se-sgRNA plasmids were confirmed by sequence analysis.

\begin{tabular}{lc} 
Table 1 & Three target sites within the basic $L F$ promoter sequences \\
\hline Score & gRNA \\
\hline 92 & CCCTACACAGGCGCTGGTACAGG \\
88 & AGTACCCCCTACACAGGCGCTGG \\
82 & AGCGCCTGTGTAGGGGGTACTGG
\end{tabular}

Note: The gRNAs are scored by inverse likelihood of offtarget binding.

\subsection{Verifying the targeting efficiency in HEK293T cells}

The human embryonic kidney HEK293T cell line was cultured in Dulbecco's modified Eagle's medium (Gibco, Grand Island, NY, USA) supplemented with $10 \%$ fetal bovine serum (Gibco, Grand Island, NY, USA) under 5\% $\mathrm{CO}_{2}$ at $37^{\circ} \mathrm{C}$. The cells were seeded into 24-well plates (Thermo Fisher Scientific, Waltham, MA, USA). After $24 \mathrm{~h}$, cells were co-transfected with a 1:1 mixture of plasmid pX330-sgRNA and plasmid 2Se-sgRNA (200 ng per well) using Lipofectamine 2000 reagent (Life Technologies, Grand Island, NY, USA). Cell fluorescence was detected by fluorescence microscopy at $24 \mathrm{~h}$ post-transfection. Mutation frequencies were counted by flow cytometry $48 \mathrm{~h}$ after transfection.

\subsection{Construction of $\mathrm{pX} 330-\mathrm{EF} 1 \alpha$-sgRNA}

Plasmid pX330 was digested with KpnI-HF and AgeI-HF (New England Biolabs, Ipswich, MA, USA) to remove the $\mathrm{CBh}$ promoter, and then the linearized $\mathrm{pX} 330$ vector was gel purified. The EF1 $\alpha$ promoter of plasmid pBudCE 4.1 (purchased from Invitrogen, Carlsbad, CA, USA) was PCR amplified using Q5 high-fidelity DNA polymerase (New England Biolabs, Ipswich, MA, USA) with a pair of primers (KpnI-EF1a-F/XmaI-EF1a-R). After electrophoresis, the PCR products were purified using the E.Z.N.A Gel Extraction Kit (Omega Bio-Tek, Norcross, GA, USA). The purified PCR products were digested by KpnI-HF and XmaI (New England Biolabs, Ipswich, MA, USA), and then ligated to the linearized pX330 vector. Finally, the construction of $\mathrm{pX} 330$-EF $1 \alpha$-sgRNA was confirmed by sequence analysis.

\subsection{Verifying the targeting efficiency of pX330-EF1 $\alpha$ - sgRNA in $\mathrm{C} 2 \mathrm{C} 12$ cells}

The C2C12 cell line was cultured in Dulbecco's modified Eagle's medium (Gibco, Grand Island, NY, USA) supplemented with $10 \%$ fetal bovine serum (Gibco, Grand Island, NY, USA) under $5 \% \mathrm{CO}_{2}$ at $37^{\circ} \mathrm{C}$. Cells were seeded into six-well plates (Thermo Fisher Scientific, Waltham, MA, USA). After $24 \mathrm{~h}$ of culture, plasmids pX330-sgRNA, pX330-EF1 $\alpha$-sgRNA and pGFP (expressing the green fluorescent protein) $(4 \mu \mathrm{g}$ per well $)$ in $150 \mu \mathrm{L}$ of electroporation medium were electro-transfected into $\mathrm{C} 2 \mathrm{C} 12$ cells using electroporation cuvettes. The cells were harvested at $48 \mathrm{~h}$ post-transfection, and genomic DNA was extracted using the DNeasy Blood \& Tissue Kit (Qiagen, Valencia, CA, USA). The genomic region flanking the CRISPR target site was PCR amplified using Q5 high-fidelity DNA polymerase (New England Biolabs, Ipswich, MA, USA) with the primer pair LF-F1-1/ LF-R1-1. After electrophoresis, PCR products were purified using the E.Z.N.A Gel Extraction Kit. A total of $400 \mathrm{ng}$ of the purified PCR products were mixed with $1 \mu \mathrm{L}$ $10 \times$ LA Buffer, and ultrapure water was added to a final volume of $10 \mu \mathrm{L}$, and subjected to a re-annealing process to enable heteroduplex formation: $95^{\circ} \mathrm{C}$ for $10 \mathrm{~min}, 95$ to $85^{\circ} \mathrm{C}$ with ramping at $-2^{\circ} \mathrm{C} / \mathrm{s}, 85^{\circ} \mathrm{C}$ for $1 \mathrm{~min}, 85$ to $75^{\circ} \mathrm{C}$ with ramping at $-0.3^{\circ} \mathrm{C} / \mathrm{s}, 75^{\circ} \mathrm{C}$ for $1 \mathrm{~min}, 75$ to $65^{\circ} \mathrm{C}$ with ramping at $-0.3^{\circ} \mathrm{C} / \mathrm{s}, 65^{\circ} \mathrm{C}$ for $1 \mathrm{~min}, 65$ to $55^{\circ} \mathrm{C}$ with ramping at $-0.3^{\circ} \mathrm{C} / \mathrm{s}, 55^{\circ} \mathrm{C}$ for $1 \mathrm{~min}, 55$ to $45^{\circ} \mathrm{C}$ with ramping at $-0.3^{\circ} \mathrm{C} / \mathrm{s}, 45^{\circ} \mathrm{C}$ for $1 \mathrm{~min}, 45$ to $35^{\circ} \mathrm{C}$ with ramping at $-0.3^{\circ} \mathrm{C} / \mathrm{s}, 35^{\circ} \mathrm{C}$ for $1 \mathrm{~min}, 35$ to $25^{\circ} \mathrm{C}$ with ramping at $-0.3^{\circ} \mathrm{C} / \mathrm{s}, 25^{\circ} \mathrm{C}$ for $1 \mathrm{~min}$, and a final hold at 
$4^{\circ} \mathrm{C}$. After annealing, $10 \mu \mathrm{L}$ of the products were treated with $2 \mu \mathrm{L}$ of NEB buffer 2 and $0.5 \mu \mathrm{L}$ T7 endonuclease 1 (T7EN1) (New England Biolabs, Ipswich, MA, USA), and $7.5 \mu \mathrm{L}$ of ultrapure water was added, resulting in a final volume of $20 \mu \mathrm{L}$. After incubation at $37^{\circ} \mathrm{C}$ for $1 \mathrm{~h}$, the products were added to $4 \mu \mathrm{L}$ of $6 \times$ loading buffer and analyzed on $8 \%$ Tris-borate-EDTA (TBE) polyacrylamide gels. Gels were stained with ethidium bromide for $5 \mathrm{~min}$ and imaged with a Gel Logic 212 Imaging System (Eastman Kodak, Rochester, NY, USA). Quantification was based on relative band intensities. The indel percentage was determined by the formula $100 \times(1-$ $\left.(1-(b+c) /(a+b+c))^{1 / 2}\right)$, wherein a is the integrated intensity of the undigested PCR product and $\mathrm{b}$ and $\mathrm{c}$ are the integrated intensities of each cleavage product.

\subsection{Pronuclear microinjection}

Kunming female mice were superovulated and mated with Kunming male mice, and fertilized eggs were collected from the oviduct. Pronuclear stage eggs were injected with pX330-EF $1 \alpha$-sgRNA at a concentration of $1 \mathrm{ng} \cdot \mu \mathrm{L}^{-1}$. The eggs were cultivated in M16 (Sigma-Aldrich, St. Louis, MO, USA) for a moment and then transferred into both sides of the fallopian tubes of pseudopregnant Kunming females.

\subsection{Detection of mutations in mice}

Twenty days after birth, genomic DNA was extracted from the tails of mice. The regions surrounding the target locus in the $L F$ gene of the postnatal mice were genotyped by PCR (the primers for the 11 mice used in the first microinjection were LF-F1-1/LF-R1-1; the primers for the 18 mice used in the second microinjection were TB-LF-F/ TB-LF-R). Then, genomic DNA was PCR amplified using Q5 high-fidelity DNA polymerase. After purifying the PCR products, a total of $400 \mathrm{ng}$ of the purified PCR products were then denatured, annealed and treated with T7EN1 nuclease. Then, the products with mutations detected by the T7EN1 assay were subcloned into the pMD-19T vector (Takara, Dalian, China), and the clones for each sample were randomly picked and sequenced.

\subsection{Plasmid integration analysis}

Four pairs of primers were randomly designed for different locations in the plasmids (cas9-ZL-F1/R1, cas9-ZL-F2/R2, cas9-ZL-F3/R3, cas9-ZL-F4/R4). To determine whether the plasmid was integrated into the mouse genome, genomic DNA isolated from the mutants was subjected to PCR amplification with the four pairs of primers.

\subsection{Off-target analysis}

To test whether there were off-target mutations, 12 potential off-target candidates were selected using CRISPR Genome Engineering Resources. We designed 12 pairs of primers (OT-LF-F1/1-OT-LF-F12/12) that were complementary to approximately $600 \mathrm{bp}$ regions of potential off-target sites. Then, the regions containing the potential off-target sites were amplified with KOD-plus high-fidelity DNA polymerase (TOYOBO, Osaka, Japan) and sequenced.

\section{Results}

\subsection{Design of sgRNA and preparation of the CRISPR/Cas9 plasmids}

The size of the mouse lactoferrin gene promoter is $7.5 \mathrm{~kb}$. The transcription level directed by the LF minimal promoter is controlled by three regions, including both positive (between nucleotides -1739 and -922) and negative (between nucleotides -2644 and -1739 , and between nucleotides -589 and -291) regulatory sequences. Moreover, the basic promoter of the mouse lactoferrin gene is located between nucleotides -234 and -21 ; deletion of this region completely abolishes its activity ${ }^{[25]}$. Thus, we designed three sgRNAs (sgRNA1-1, sgRNA1-2, sgRNA1-3) targeting this critical area (Fig. 1a), and completed the construction of three pX330sgRNA and three $2 \mathrm{Se}$-sgRNA plasmids. To validate which sgRNA sequence works, a dual-color reporter vector (2Se) was used to indicate the activity of RNA-guided hSpCas9. The reporter comprises a monomeric red fluorescent protein $(m R F P)$ gene, an RNA-guided $\mathrm{hSpCas} 9$ target sequence, and one enhanced GFP $(e G F P)$ gene. The reporter-transfected cells will express only mRFP in the absence of hSpCas9 nuclease activity; in the presence of nuclease activity, the transfected cells will express both mRFP and eGFP ${ }^{[26]}$. To select the best pX330-sgRNA, we co-transfected pX330-sgRNA and 2Se-sgRNA into HEK293T cells. Twenty-four hours after the transfection, most of the cells expressed mRFP. Only a small fraction of cells expressed both mRFP and eGFP, suggesting that RNA-guided hSpCas9 indeed worked (Fig. 1b). Fortyeight hours after the transfection, the mutation frequencies of the three targets (sgRNA1-1, sgRNA1-2, sgRNA1-3) were $28.6 \%, 26.0 \%$, and $22.0 \%$, respectively, as assessed by flow cytometry (Fig. 1c).

The promoter of hSpCas 9 is $\mathrm{CBh}$ in the initial pX330 plasmid. We replaced the $\mathrm{CBh}$ promoter with the EF1 $\alpha$ promoter, which is constitutively expressed in mouse cells, to improve the expression efficiency of hSpCas $9^{[27]}$. This promoter replacement was applied to pX330-sgRNA1-1, which had the highest targeting efficiency. To verify that the new plasmid, pX330-EF1 $\alpha$-sgRNA1-1, could still play a role, we transfected pX330-sgRNA1-1, pX330-EF1 $\alpha$ sgRNA1-1, and pGFP into mouse $\mathrm{C} 2 \mathrm{C} 12$ cells. Then, we isolated genomic DNA and performed a T7EN1 assay. 
Apparent cleavages of bands were found in $\mathrm{pX} 330-\mathrm{EF} 1 \alpha-$ sgRNA1-1-transfected cells (Fig. 1d), indicating that it cleaved the target locus efficiently.

\subsection{One-step generation of $L F$ gene promoter mutant mice} by pronuclear injection of circular plasmid

Following validation of the pX330-EF1 $\alpha$-sgRNA1-1 plasmids in mouse $\mathrm{C} 2 \mathrm{C} 12$ cells, we directly injected it into the pronucleus of fertilized eggs twice. The two microinjections are summarized in Table 2. The regions surrounding the target locus in the $L F$ gene of postnatal mice were genotyped by PCR (Appendix B, Fig. S1). The PCR results demonstrated that the CRISPR/Cas9 system did not produce long insertions or deletions and the T7EN1 assay was performed to assess the targeted cleavage efficiency (Fig. 2a, Fig. 2b). Clear cleavage bands were detected in three mice born after the first microinjection and four mice born after the second microinjection. To confirm this gene mutation, subsequent sequence analysis (Fig. 2c) showed that the seven mice had $L F$ gene promoter mutations at the target site (three mice from the second microinjection) or $40 \mathrm{bp}$ upstream of the target locus (three mice from the first microinjection) or both (one mouse from the second microinjection).

\subsection{Plasmid integration and off-target analysis}

An injected plasmid may integrate into the gene target in the mouse genome. To determine whether the plasmid integrated, four pairs of primers were randomly designed

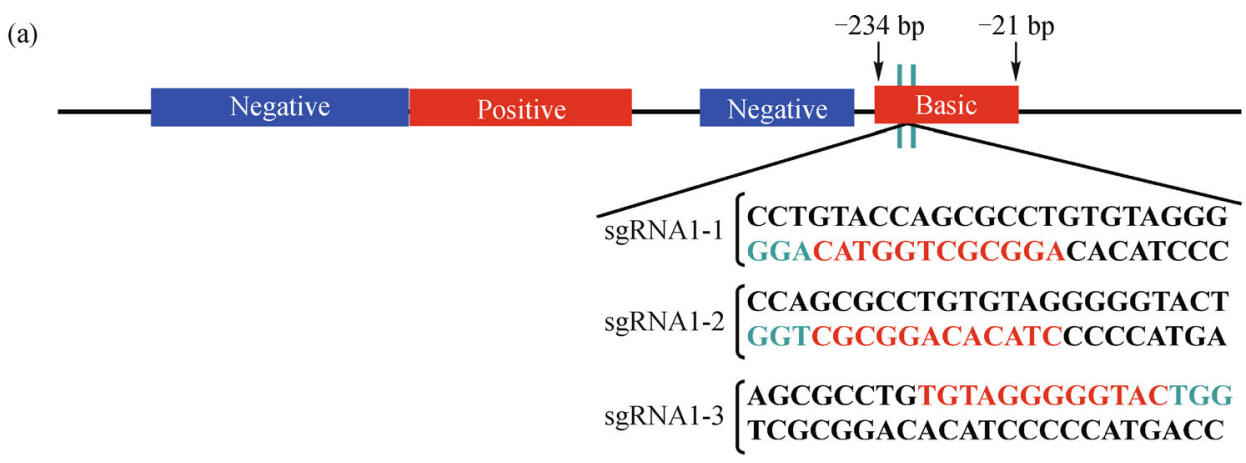

(b)

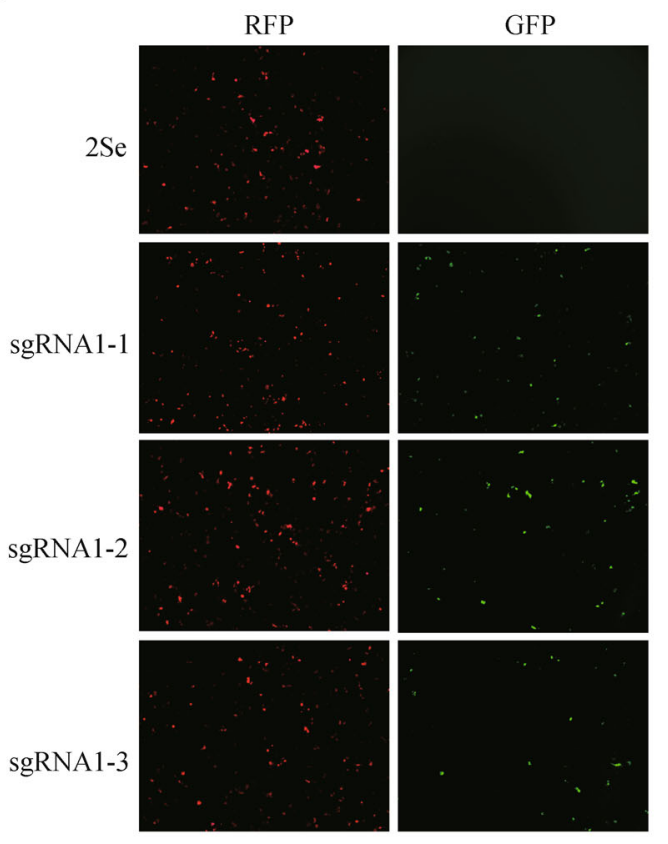

(c)
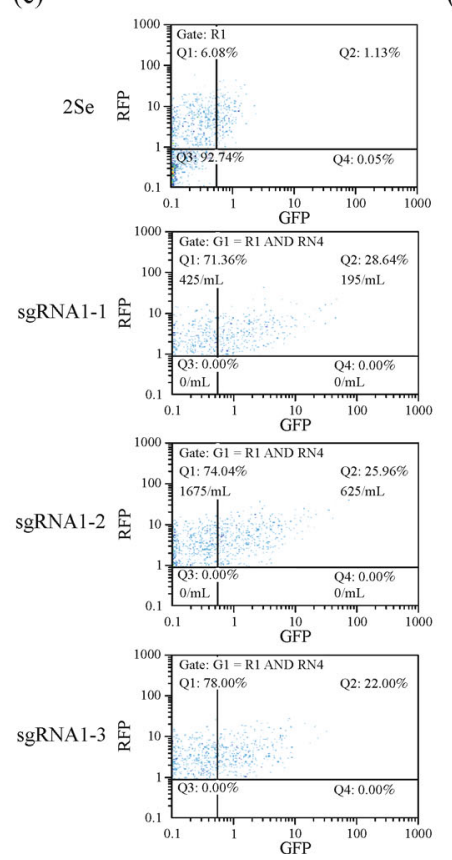

(d)

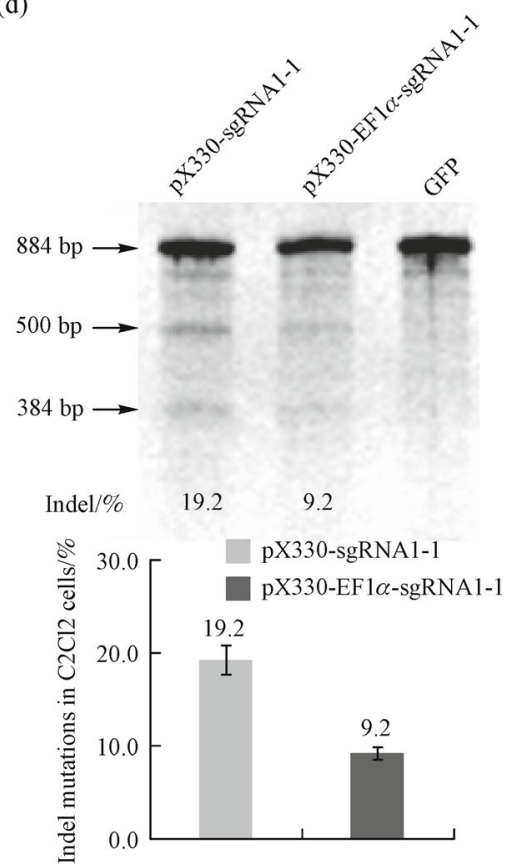

Fig. 1 Preparation of the CRISPR/Cas9 plasmids. (a) Schematic of sgRNAs targeting sites at critical regions of the $L F$ promoter. The protospacer adjacent motif (PAM) sequence is labeled in blue and a 12 bp seed sequence is highlighted in red; (b) observed expression of red fluorescent protein (RFP) and green fluorescent protein (GFP), $24 \mathrm{~h}$ after co-transfection of pX330-sgRNA and 2Se-sgRNA, by fluorescence microscopy; (c) the percentage of $\mathrm{RFP}^{+} \mathrm{GFP}^{+}$cells by flow cytometry $48 \mathrm{~h}$ after co-transfection is shown; (d) T7EN1 assay for Cas9-mediated cleavage in $\mathrm{C} 2 \mathrm{C} 12$ cells and its densitometry analysis by Image J. 
Table 2 Generation of mutant mice via pX330 plasmid injection

\begin{tabular}{lcccccc}
\hline No. & Injected & Pregnancy & Newborn Mutation & Indel mutation frequency & Mutation type \\
\hline 1 & 238 & 4 & 11 & 3 & $3 / 11(27 \%)$ & $\begin{array}{c}\text { Three mice were all mutated } 40 \text { bp upstream of the target locus } \\
2\end{array}$ \\
325 & 5 & 18 & 4 & $4 / 18(22 \%)$ & $\begin{array}{c}\text { Three of four were mutated at the target site, and the other one was mutated at } \\
\text { both the target site and } 40 \text { bp upstream of the target locus }\end{array}$ \\
\hline
\end{tabular}

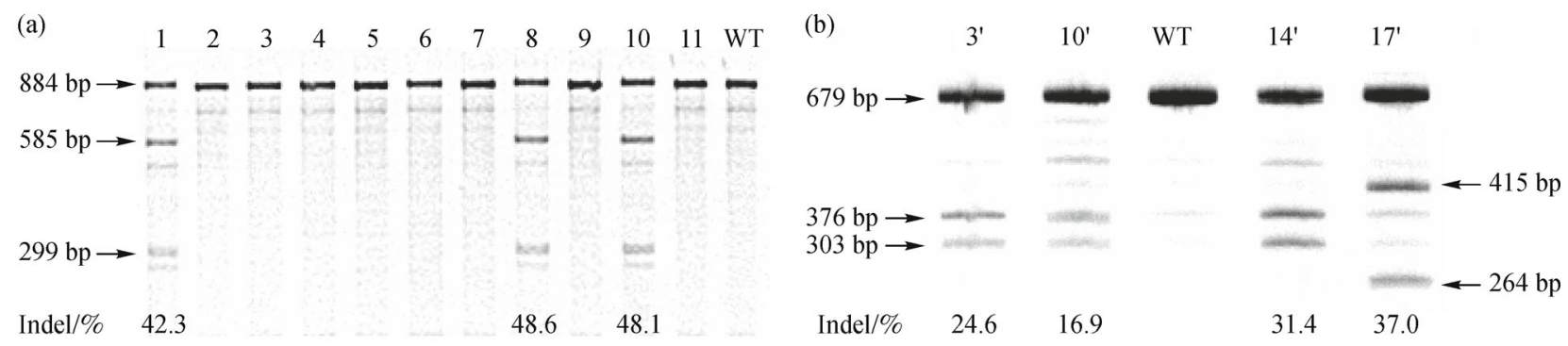

(c) 5' G ATCCTGCAG-//-G-//-CCT-GTACCAGCGCCTGTGTAGG 3'

WT

5' GACCCTGCAG-//-G-//-CCT-GTACCAGCGCCTGTGTAGG 3' GATG

5' G ATCCTGCAG-//-G-//-CCT-GTACCAGCACCTGTGTAGG 3'

5' G ATCCTGCAG-//-G-//-ATT - TA - - GCGCCTGTGTAGG 3'

5' G ATCGTGCAT-//-T-//-CCTGTACGTACCCCTGTGTGGG 3'

$\begin{array}{ll}(+4 \mathrm{bp}, \mathrm{ml}) & {[\times 3]} \\ (\mathrm{ml}) & {[\times 2]} \\ (-4 \mathrm{bp}, \mathrm{m} 2) & {[\times 1]} \\ (\mathrm{m} 8) & {[\times 1]}\end{array}$

Fig. 2 Generation of $L F$ gene promoter mutation mice via the CRISPR/Cas9 system. (a) T7EN1 assay for Cas9-mediated cleavage in newborn mice from the first microinjection. Four pregnant mice resulting from the first microinjection gave birth to 11 mice. WT is the genome of Kunming wild-type mice; (b) T7EN1 assay for Cas9-mediated cleavage in newborn mice from the second microinjection. 3', $10^{\prime}, 14^{\prime}$, and $17^{\prime}$ represent the genomes of the newborn mice from the second microinjection; (c) DNA sequences of the WT and four mutant alleles in seven mice. The target site is underlined, and the PAM sequence is labeled in green. -//- represents an omitted base, and 18 and $15 \mathrm{bp}$ are omitted from left to right. The numbers of mutant mice are in brackets.

in different locations of the plasmid. Genomic DNA, isolated from the seven mice generated by CRISPR/Cas9, was subjected to PCR amplification with the four pairs of primers. The final PCR results showed that the plasmid did not integrate into the mouse genome at this level of detection (Appendix B, Fig. S2).

It is known that a small number of mismatches between sgRNAs and the target DNA can be tolerated, and one to four base-pair mismatches did not affect the ability of sgRNAs to identify their potential targets ${ }^{[28,29]}$. To test whether there were off-target mutations in these genetically modified mice, we searched for off-target sites in the mouse genome containing up to three or four base-pair mismatches compared with the $20 \mathrm{bp}$ sgRNA coding sequence. We predicted 12 potential off target candidates, then the approximate $600 \mathrm{bp}$ regions containing the potential off-target sites were amplified and sequenced. No mutations were observed at these 12 hypothetical offtarget loci in any of the seven gene-targeted mice (Table 3).

\section{Discussion}

In this study, we used CRISPR/Cas-mediated genome editing and obtained mice genetically modified in the $L F$ promoter by injecting plasmid pX330, which expresses sgRNA and hSpCas9, into fertilized eggs of mice. The mutation efficiency was $24 \%$ (7/29), which indicates that zygote injection of circular plasmid is a viable and efficient method for generating mouse models with gene modification. Previously, the generation of gene-targeted animals via the CRISPR/Cas9 system was realized through microinjection of a Cas9 RNA:sgRNA mixture into fertilized eggs. Instead, in this study, we avoided the complex preparation, careful handling and storage of RNA, and injected plasmid pX330 expressing sgRNA and hSpCas9 instead of RNA. Differences have been found when using mRNA and plasmids to generate mutant mice via pronuclear injection of circular plasmid ${ }^{[30]}$ and injecting exogenous components may have an adverse effect on mice. To minimize such a risk, we used a circular plasmid to reduce plasmid integration into the mouse genome. Moreover, we also analyzed plasmid integration in the mouse genome, and the results showed that the injected plasmid did not integrate into the genome. In addition, although prolonged existence of plasmid may increase the chance of off-target mutations, transient expression by pronuclear injection could decrease this risk. 
Table 3 Off-target analysis in $L F$ mutant mice

\begin{tabular}{llcl}
\hline Site name & \multicolumn{1}{c}{ Sequence } & $\begin{array}{c}\text { Indel mutation frequency } \\
\text { (Mutant/Total) }\end{array}$ & \multicolumn{1}{c}{ Coordinate } \\
\hline Target site & CCCTACACAGGCGCTGGTACAGG & $/$ & chr 9: 111019095-111019117 \\
OT1 & aCaTACtCAGGCtCTGGTACAGG & $0 / 7$ & chr 19: $48087277-48087299$ \\
OT2 & CtgTACACAGGtGCTGGTAaTGG & $0 / 7$ & chr 18: 35579256-35579278 \\
OT3 & tCCTgCACAGGCtCTGGTAtAGG & $0 / 7$ & chr 2: 121955005-121955027 \\
OT4 & tCCTgCACAGGCtCTGGTAtAGG & $0 / 7$ & chr X: 91332097-91332119 \\
OT5 & tCCTgCACAGGCtCTGGTAtAGG & $0 / 3$ & chr X: 92015969-92015991 \\
OT6 & CCacACACAGGgaCTGGTACAGG & $0 / 3$ & chr 4: 135896156-135896178 \\
OT7 & CaCTACACAGGaGCTtGTACTGG & $0 / 3$ & chr 7: 107035334-107035356 \\
OT8 & CCCTgCgCtGGCcCTGGTACAGG & $0 / 3$ & chr 3: 27673749-27673771 \\
OT9 & CCCcACACAaGCtCTGcTACTGG & $0 / 3$ & chr 10: 111084383-111084405 \\
OT10 & CCCcAaACAGGaGCTGGTAgGGG & $0 / 3$ & chr X: 134240517-134240539 \\
OT11 & CCCTttACAGtCcCTGGTACAGG & $0 / 3$ & chr 1: 166363559-166363581 \\
OT12 & CCCTACAtAGatGCTGtTACAGG & $0 / 3$ & chr 19: 48586214-48586236 \\
\hline
\end{tabular}

Note: + , sense strand; - , antisense strand. Mismatches from the on-target sequence are lower-case and boldfaced.

The CRISPR/Cas9 system makes rapid, simple and costeffective gene mutations, but the high off-target mutation rate cannot be ignored. Previous reports have suggested that one to four base-pair mismatches will not affect the ability of sgRNAs to identify their potential targets. This degree of mismatch reduces, rather than completely preventing, Cas9 cleavage, which leads to a high frequency of off-target mutations ${ }^{[28,29]}$. We analyzed the off-target rate of seven genetically modified mice, and no mutations were detected in 12 potential off-target sites. However, with the optimization of the CRISPR/Cas9 system, there are also solutions to improve CRISPR-Cas nuclease specificity, for example, using truncated sgRNAs (17 or $18 \mathrm{bp})^{[31]}$, double-nick ${ }^{[32]}$ and dimeric CRISPR RNAguided FokI nucleases ${ }^{[33]}$.

The CRISPR/Cas9 system mediated gene mutations at the target sites in previous reports ${ }^{[28,34]}$, but it also mediated gene mutations at both the target site and $40 \mathrm{bp}$ upstream of the target locus in our study. In the first microinjection, we obtained three mice that had a $4 \mathrm{bp}$ insertion $40 \mathrm{bp}$ upstream of the target locus, and in the second microinjection, we still generated one mouse with a mutation $40 \mathrm{bp}$ upstream of the designated target locus. The reason for this is more than likely to be due to the structure of the $L F$ gene promoter. Furthermore, doublestranded break and non-homologous end joining mediated by the CRISPR/Cas9 system may occur at non-target sites.

\section{Conclusions}

We have successfully obtained $L F$ gene promoter mutation mice by injecting plasmid pX330 expressing Cas9/gRNA complexes into zygotes without detectable random plas- mid integration and off-target mutations. This simple and quick gene editing system will have many potential applications for generating genetically modified small animal models.

Acknowledgements This study was supported by the Modern Agroindustry Technology Research Systems of China (CARAS-37) and the Domain Specific Projects for Transgenic Breeding (2014ZX08010004-008)

Supplementary materials The online version of this article at http://dx.doi. org/10.15302/J-FASE-2015059 contains supplementary material (Appendix $\mathrm{A}$ and $\mathrm{B})$.

Compliance with ethics guidelines Mengxu Ge, Fei Liu, Fei Chang, Zhaolin Sun, Jing Fei, Ying Guo, Yunping Dai, Zhengquan Yu, Yaofeng Zhao, Ning Li and Qingyong Meng declare that they have no conflict of interest or financial conflicts to disclose.

All applicable institutional and national guidelines for the care and use of animals were followed.

\section{References}

1. Sorensen M, Sorensen S. The proteins in whey. Compte rendu des Travaux du Laboratoire de Carlsberg Ser Chim, 1940, 23(7): 55-99

2. Groves M L. The isolation of a red protein from milk. Journal of the American Chemical Society, 1960, 82(13): 3345-3350

3. Johanson B, Virtanen A I, Tweit R C, Dodson R M. Isolation of an iron-containing red protein from human milk. Acta Chemica Scandinavica, 1960, 14(2): 510-512

4. Montreuil J, Tonnelat J, Mullet S. Preparation and properties of lactosiderophilin (lactotransferrin) of human milk. Biochimica et Biophysica Acta, 1960, 45: 413-421

5. Actor J K, Hwang S A, Kruzel M L. Lactoferrin as a natural immune modulator. Current Pharmaceutical Design, 2009, 15(17): 19561973 
6. Ward P P, Conneely O M. Lactoferrin: role in iron homeostasis and host defense against microbial infection. Biometals, 2004, 17(3): 203-208

7. Blais A, Malet A, Mikogami T, Martin-Rouas C, Tomé D. Oral bovine lactoferrin improves bone status of ovariectomized mice. American Journal of Physiology, Endocrinology and Metabolism, 2009, 296(6): E1281-E1288

8. Malet A, Bournaud E, Lan A, Mikogami T, Tomé D, Blais A. Bovine lactoferrin improves bone status of ovariectomized mice via immune function modulation. Bone, 2011, 48(5): 1028-1035

9. Mulder A M, Connellan P A, Oliver C J, Morris C A, Stevenson L M. Bovine lactoferrin supplementation supports immune and antioxidant status in healthy human males. Nutrition Research, 2008, 28(9): 583-589

10. Wang Y Z, Shan T Z, Xu Z R, Feng J, Wang Z Q. Effects of the lactoferrin (LF) on the growth performance, intestinal microflora and morphology of weanling pigs. Animal Feed Science and Technology, 2007, 135(3): 263-272

11. Velusamy S K, Ganeshnarayan K, Markowitz K, Schreiner H, Furgang D, Fine D H, Velliyagounder K. Lactoferrin knockout mice demonstrates greater susceptibility to Aggregatibacter actinomycetemcomitans-induced periodontal disease. Journal of Periodontology, 2013, 84(11): 1690-1701

12. van der Strate B W, Beljaars L, Molema G, Harmsen M C, Meijer D K. Antiviral activities of lactoferrin. Antiviral Research, 2001, 52 (3): 225-239

13. Farnaud S, Evans R W. Lactoferrin - a multifunctional protein with antimicrobial properties. Molecular Immunology, 2003, 40(7): 395405

14. Yang N, Strøm M B, Mekonnen S M, Svendsen J S, Rekdal O. The effects of shortening lactoferrin derived peptides against tumour cells, bacteria and normal human cells. Journal of Peptide Science, 2004, 10(1): 37-46

15. Garneau J E, Dupuis M, Villion M, Romero D A, Barrangou R, Boyaval P, Fremaux C, Horvath P, Magadán A H, Moineau S. The CRISPR/Cas bacterial immune system cleaves bacteriophage and plasmid DNA. Nature, 2010, 468(7320): 67-71

16. Gasiunas G, Barrangou R, Horvath P, Siksnys V. Cas9-crRNA ribonucleoprotein complex mediates specific DNA cleavage for adaptive immunity in bacteria. Proceedings of the National Academy of Sciences of the United States of America, 2012, 109 (39): E2579-E2586

17. Jinek M, Chylinski K, Fonfara I, Hauer M, Doudna J A, Charpentier E. A programmable dual-RNA-guided DNA endonuclease in adaptive bacterial immunity. Science, 2012, 337(6096): 816-821

18. Mussolino C, Cathomen T. RNA guides genome engineering. Nature Biotechnology, 2013, 31(3): 208-209

19. Cho S W, Kim S, Kim J M, Kim J S. Targeted genome engineering in human cells with the Cas9 RNA-guided endonuclease. Nature Biotechnology, 2013, 31(3): 230-232

20. Shen B, Zhang J, Wu H, Wang J, Ma K, Li Z, Zhang X, Zhang P, Huang X. Generation of gene-modified mice via Cas9/RNAmediated gene targeting. Cell Research, 2013, 23(5): 720-723

21. Hwang W Y, Fu Y, Reyon D, Maeder M L, Tsai S Q, Sander J D,
Peterson R T, Yeh J R, Joung J K. Efficient genome editing in zebrafish using a CRISPR-Cas system. Nature Biotechnology, 2013, 31(3): 227-229

22. Li W, Teng F, Li T, Zhou Q. Simultaneous generation and germline transmission of multiple gene mutations in rat using CRISPR-Cas systems. Nature Biotechnology, 2013, 31(8): 684-686

23. Niu Y, Shen B, Cui Y, Chen Y, Wang J, Wang L, Kang Y, Zhao X, Si W, Li W, Xiang A P, Zhou J, Guo X, Bi Y, Si C, Hu B, Dong G, Wang H, Zhou Z, Li T, Tan T, Pu X, Wang F, Ji S, Zhou Q, Huang $\mathrm{X}$, Ji W, Sha J. Generation of gene-modified cynomolgus monkey via Cas9/RNA-mediated gene targeting in one-cell embryos. Cell, 2014, 156(4): 836-843

24. Han H, Ma Y, Wang T, Lian L, Tian X, Hu R, Deng S, Li K, Wang F, Li N, Liu G, Zhao Y, Lian Z. One-step generation of myostatin gene knockout sheep via the CRISPR/Cas9 system. Frontiers of Agricultural Science and Engineering, 2014, 1(1): 2-5

25. Liu Y H, Teng C T. Characterization of estrogen-responsive mouse lactoferrin promoter. Journal of Biological Chemistry, 1991, 266 (32): 21880-21885

26. Ramakrishna S, Cho S W, Kim S, Song M, Gopalappa R, Kim J S, Kim H. Surrogate reporter-based enrichment of cells containing RNA-guided Cas9 nuclease-induced mutations. Nature Communications, 2014, 5(3378): 3378

27. Dejosez M, Krumenacker J S, Zitur L J, Passeri M, Chu L F, Songyang Z, Thomson J A, Zwaka T P. Ronin is essential for embryogenesis and the pluripotency of mouse embryonic stem cells. Cell, 2008, 133(7): 1162-1174

28. Yang H, Wang H, Shivalila C S, Cheng A W, Shi L, Jaenisch R. One-step generation of mice carrying reporter and conditional alleles by CRISPR/Cas-mediated genome engineering. Cell, 2013, 154(6): 1370-1379

29. Cong L, Ran F A, Cox D, Lin S, Barretto R, Habib N, Hsu P D, Wu X, Jiang W, Marraffini L A, Zhang F. Multiplex genome engineering using CRISPR/Cas systems. Science, 2013, 339 (6121): 819-823

30. Mashiko D, Fujihara Y, Satouh Y, Miyata H, Isotani A, Ikawa M. Generation of mutant mice by pronuclear injection of circular plasmid expressing Cas9 and single guided RNA. Scientific Reports, 2013, 3(3355): 3355

31. Fu Y, Sander J D, Reyon D, Cascio V M, Joung J K. Improving CRISPR-Cas nuclease specificity using truncated guide RNAs. Nature Biotechnology, 2014, 32(3): 279-284

32. Ran F A, Hsu P D, Lin C Y, Gootenberg J S, Konermann S, Trevino A E, Scott D A, Inoue A, Matoba S, Zhang Y, Zhang F. Double nicking by RNA-guided CRISPR Cas9 for enhanced genome editing specificity. Cell, 2013, 154(6): 1380-1389

33. Tsai S Q, Wyvekens N, Khayter C, Foden J A, Thapar V, Reyon D, Goodwin M J, Aryee M J, Joung J K. Dimeric CRISPR RNAguided FokI nucleases for highly specific genome editing. Nature Biotechnology, 2014, 32(6): 569-576

34. Wang H, Yang H, Shivalila C S, Dawlaty M M, Cheng A W, Zhang $\mathrm{F}$, Jaenisch R. One-step generation of mice carrying mutations in multiple genes by CRISPR/Cas-mediated genome engineering. Cell, 2013, 153(4): 910-918 